\title{
SYMPA, a dedicated instrument for Jovian seismology
}

\section{Principle and performance}

\author{
F. X. Schmider ${ }^{1}$, J. Gay ${ }^{2}$, P. Gaulme ${ }^{1}$, C. Jacob ${ }^{1}$, L. Abe ${ }^{1}$, M. Alvarez ${ }^{3}$, J. A. Belmonte ${ }^{4}$, E. Fossat ${ }^{1}$, B. Gelly ${ }^{5}$, \\ T. Guillot ${ }^{2}$, F. Jeanneaux ${ }^{1}$, B. Mosser ${ }^{6}$, and J.-C. Valtier ${ }^{2}$ \\ 1 Université de Nice Sophia-Antipolis, Laboratoire Universitaire d'Astrophysique de Nice, 06108 Nice Cedex 2, France \\ e-mail: schmider@unice.fr \\ 2 Observatoire de la Côte d'Azur, 06034 Nice Cedex 1, France \\ 3 Observatorio Astronómico Nacional, Instituto de Astronomía, Universidad Nacional Autónoma de México, Apto. Postal 877, \\ Ensenada, B.C., México \\ ${ }^{4}$ Instituto de Astrofísica de Canarias, Tenerife, Spain \\ 5 THEMIS Observatory, La Laguna, Tenerife, Spain \\ ${ }^{6}$ Observatoire de Paris-Meudon, LESIA, Meudon, France
}

Received 22 December 2006 / Accepted 21 June 2007

\section{ABSTRACT}

\begin{abstract}
Aims. The detection and measurement of acoustic modes on the giant planets of the solar system is of great interest for the study of the internal structure and evolution of the giant planets, as well as the study of the solar system formation. Such observations require a dedicated instrument and observing procedure.

Methods. We describe the principle and the performance of an instrument dedicated to seismology of giant planets. In this first paper, we describe the principle and the optical scheme, and derive the theoretical performances. As for the Sun, it is possible to measure modes with spatial resolution, but a larger collecting area is necessary. As for asteroseismology in general, continuity in the observation is also required.

Results. From results obtained at the laboratory, we derive the actual performance of the instrument and estimate its capabilities in network observations.

Conclusions. We demonstrate that the proposed instrument and strategy is adapted for the seismology of giant planets. In a second paper, we will present the first data set obtained with it, explain the data reduction procedure, and present preliminary results.
\end{abstract}

Key words. instrumentation: interferometers - instrumentation: spectrographs - techniques: radial velocities - stars: oscillations planets and satellites: individual: Jupiter - Sun: helioseismology

\section{Introduction}

Since the very beginning of helioseismology, in 1975, Jupiter was identified as an interesting target for seismic study, first theoretically (Vorontsov et al. 1976), then observationally. In 1987, Jupiter was observed by Deming (Deming et al. 1989), who was looking for thermal variation in mid-infrared images, without success. Simultaneously, Schmider et al. (1991) obtained 6 consecutive nights of data, with a sodium cell instrument designed for radial velocity measurement. The Fourier spectrum of the global signal exhibited a power excess in the [1, $2 \mathrm{mHz}]$ frequency range, that could not be explained by low frequency noise. A careful analysis of the power spectrum density revealed the signature of frequency doublets separated by $56 \mu \mathrm{Hz}$, as expected from the rotational splitting due the rotation of Jupiter with a period of $10 \mathrm{~h}$.

In 1991, 1993 and 1998, other observations were conducted by Philippe Delache, Jean Gay and Benoit Mosser (Mosser et al. 1993, 2000) with the use of the FTS at the CFHT. Although they were looking for different degree modes (differential measurement between north and south hemisphere), they found similar power excess and again the signature of the rotational splitting and of the large separation around $142 \mu \mathrm{Hz}$, already found in the observations of Schmider (Schmider et al. 1991;
Mosser et al. 1991) and interpreted as the signature of the main Jovian harmonics.

The interpretation of the eigenmodes remained difficult, due to the limited duration of the time series, hence the limited frequency resolution, and to the many sidelobes. However, these observations have shown the potential of asteroseismology in order to study the internal structure of Jupiter.

Many authors have then emphasized the importance of the seismic constraints in order to improve our knowledge of the internal structure of Jupiter, as for the Sun (Guillot et al. 1995; Gudkova et al. 1999). Indeed, a lot of Jovian internal quantities remain unknown: the amount and repartition of heavy elements, a precise equation of state, the existence of a plasma phase transition between metallic and molecular hydrogen. The measurement and interpretation of Jovian oscillation will have a lot of consequences in high-pressure physics, on theories of solar system formation (where the role of Jupiter was essential), and for comparison with evolution of other planetary systems.

These observations also proved that giant planet seismic observations require special procedure, implying continuity (as for asteroseismology in general) but also angular resolution. Unlike stars, the giant planets of the solar system, and in particular Jupiter and Saturn, can be spatially resolved with a moderate size telescope through the atmospheric turbulence. It appears then essential to take advantage of this possibility, both to identify 
directly the geometry of the observed modes, and to detect as many modes as possible. As shown by Gudkova (1999), a detection of modes up to $\ell=25$ would allow the study of the plasma phase transition, if any. Moreover, the sensitivity of Doppler shift measurements is dramatically reduced when the spectral lines are broadened by the fast rotation of the target, as it is the case for Jupiter and Saturn. Spatially resolved spectrometric measurements will not suffer from this effect and will be more sensitive than the previous unresolved observations.

In the present paper, we present a new instrumental concept, derived from previous experiments, and responding to the requirements of giant planet seismology. We describe the instrumental principle and we estimate the theoretical performance of observations obtained with a network of three copies of such instruments, and report the laboratory measurements of a first prototype. In conclusion, we show that this instrument is perfectly suited for the goal of giant planet seismology, and give some prospects of evolution of this new field.

\section{The SYMPA instrument}

These scientific requirements define an observation strategy and suggest the principle and design of an optimised instrument. The continuity in the observations is partially obtained by simultaneous observations in sites at different longitudes, like helioseismology networks. Specific networks have been operated for different kinds of stars. STEPHI (Michel et al. 2000), dedicated to $\delta$ Scuti stars, has proved its efficiency. This implies the use of three copies of the same instrument at the three sites.

An angular resolution, as high as possible, is required to monitor the highest possible modes. Both previously used concepts, magnetic optical filter or Fourier Transform Spectrometer, offer the capacity of high resolution imaging. Both have been used in different instrument for helioseismology with angular resolution: LOW-L (Tomczyk et al. 1995) or GONG (Harvey et al. 1996). However, the FTS type offers a larger velocity working range, and is therefore preferred for giant planet seismology, because of the velocity shift induced by the Earth orbital movement.

As the Fourier Transform Spectrometer used for the observations at the CFHT (Maillard \& Michel 1982), the instrument called SYMPA (Seismographic Imaging Interferometer for Monitoring of Planetary Atmospheres) is based on the properties of an interferometer to produce at the output the Fourier transform of the incoming spectrum when the Optical Path Difference (OPD) changes. In absence of any spectral lines, the Fourier transform of a spectrum produced by a filter with a bandwidth of a few nanometers would decrease exponentially after an OPD of the same order. The presence of spectral lines randomly distributed within the input filter results in a slower decrease of the amplitude of the Fourier transform (see Fig. 1). As a consequence, the contrast of the interference fringes remains large enough even for an OPD of several centimeters. On the other hand, the Doppler shift of these lines, due to velocity variations at the surface of Jupiter, induces a phase shift in the interference pattern, proportional to the OPD. At high values of the OPD, this phase shift becomes measurable, and an optimal value of the OPD can be found.

Indeed, for a given spectrum $S_{\mathrm{p}}(\sigma)$, the fringe pattern obtained by varying the OPD $\Delta$ can be written as

$$
\begin{aligned}
I(\Delta) & =\left|\int S_{\mathrm{p}}(\sigma) \mathrm{e}^{2 \pi \sigma \Delta} \mathrm{d} \sigma\right|^{2} \\
& \simeq I_{0}\left[1+C(\Delta) \cos \left(2 \pi \sigma_{0} \Delta+\varphi_{0}\right)\right]
\end{aligned}
$$
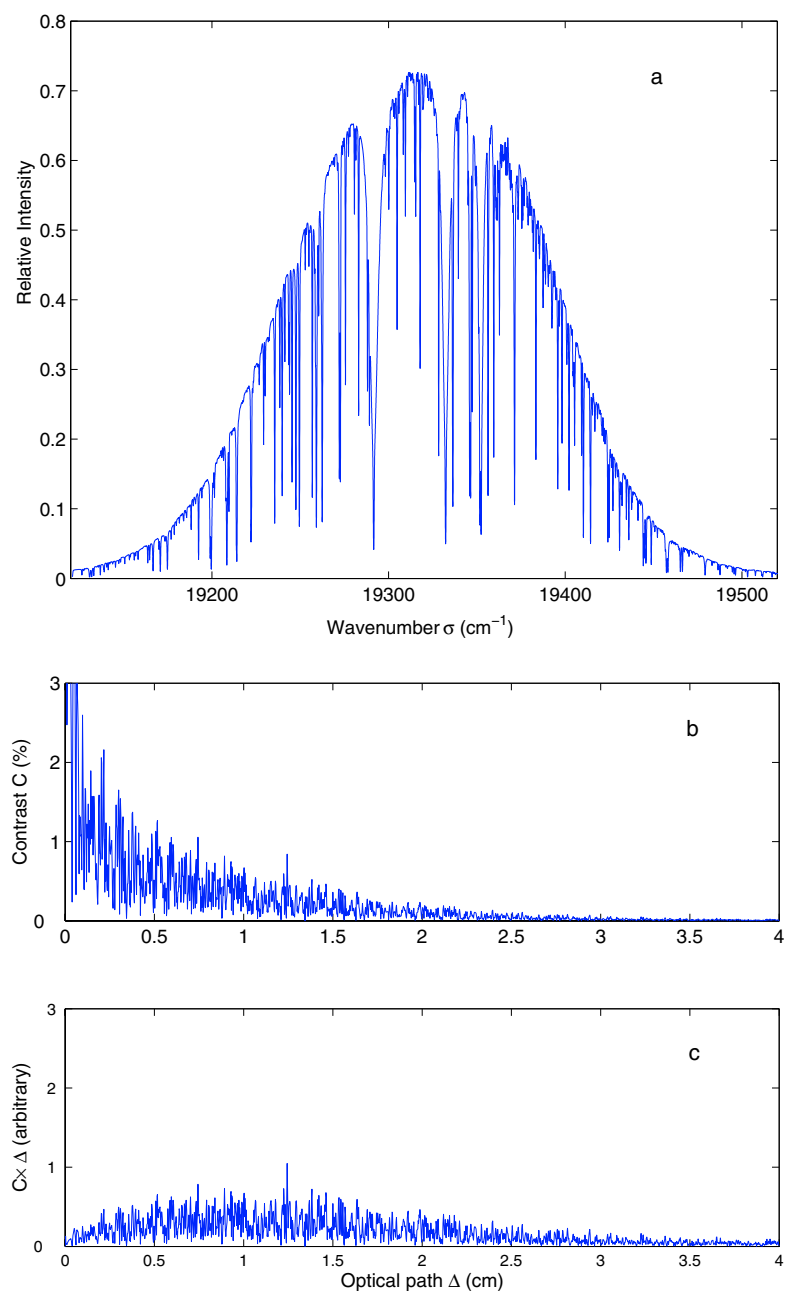

Fig. 1. a) Solar spectrum reflected at the surface of Jupiter, through a filter centered on the magnesium triplet at $517 \mathrm{~nm}\left(19340 \mathrm{~cm}^{-1}\right)$. b) Square modulus of the Fourier transform of the spectrum in a), as a function of the optical path difference $\Delta$, in $\mathrm{cm}(\Delta$ is the conjugated variable of the wave number $\sigma$ ). It gives the contrast $\mathrm{C}$ of the interference fringes. c) The same curve multiplied by the OPD $\Delta$. This value is proportional to the sensitivity of the phase of the fringes to the radial velocity. It shows a clear maximum of sensitivity for an OPD of $1.24 \mathrm{~cm}$.

on a small interval of $\Delta$, where $C$ is the slowly varying amplitude of the fringes, $\sigma_{0}$ the central wave number of the bandwidth, and $\varphi_{0}$ an arbitrary phase shift.

Therefore, a Doppler shift of the spectrum by a quantity

$\delta \sigma=-\sigma_{0} \frac{\delta v}{c}$

produces a phase shift

$\varphi_{1}-\varphi_{0}=2 \pi \Delta \sigma_{0} \frac{\delta v}{c}$

in the fringe pattern. The monitoring of the phase of the interference fringe allows the accurate measurement of the velocity field variations.

In order to monitor the phase of the fringe without contamination by the photometric variations, it has been classically considered to measure four points in quadrature along the fringe, by modulating the OPD periodically (Shao \& Staelin 1980). The phase is then estimated by the algorithm

$\tan (\varphi)=(A-C) /(B-D)$ 


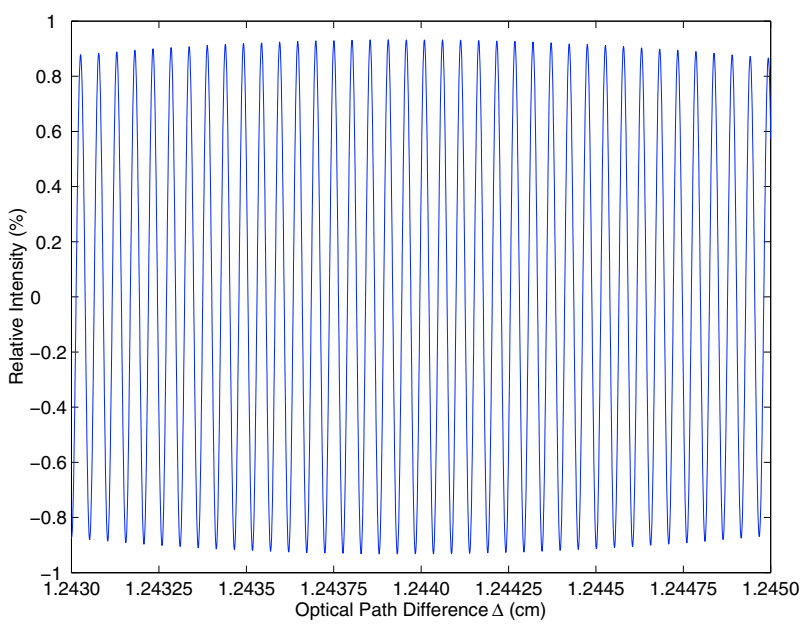

Fig. 2. Detail of the relative intensity of the fringe of the previous figure, at $1.24 \mathrm{~cm}$. The contrast of the fringe remains almost uniform for a range of OPD of several tenth of microns.

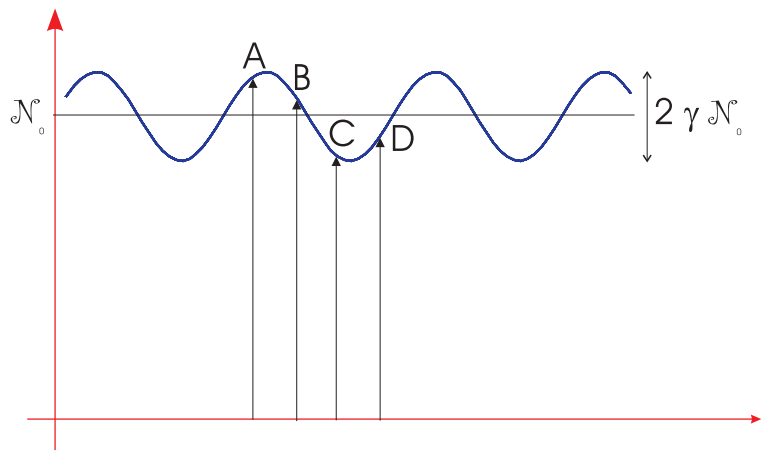

Fig. 3. The phase of the fringe can be derived from the intensities measured in four points along a period. The contrast $\gamma$, defined as $\left(I_{\max }-I_{\min }\right) /\left(I_{\max }+I_{\min }\right)$, is the mean amplitude of the fringes at the considered OPD, and $\mathcal{N}_{0}$ is the average photon number in each channel.

where $A, B, C$, and $D$ are the relative intensities at the four points separated by $\pi / 2$ in phase (see Fig. 3). This principle has also been used in several helioseismographs, like GONG or MDI.

Here we propose a simple instrument to monitor the phase variations. Instead of modulating the OPD, four images almost in quadrature are produced simultaneously. The relative photometric measurement of the four images allows us to recover the phase of the interference pattern at each point of the image, in order to calculate a velocity map (Schmider et al. 2003; Mosser et al. 2003). The quadrature of the four images is obtained here by a metallic reflection on one arm of the Mach-Zehnder interferometer, when the other arm presents a total reflection. The effect is to introduce a phase shift of about $90^{\circ}$ between one polarization and the other. By construction, the interferometer has two outputs opposite in phase. After a separation of the two polarizations, we finally obtain four images separated by $90^{\circ}$ along one fringe period (see Fig. 3).

\subsection{Choice of the bandwidth}

The signal-to-noise ratio of the velocity measurement is linked to the product of the contrast $\gamma$ by the OPD $\Delta$. As the contrast vanishes approximately as $\exp (-\Delta / \beta)$, where $\beta$ is the inverse of the linewidths in $\mathrm{cm}^{-1}$, the product $\gamma \times \Delta$ is maximum when $\Delta$ is of the order of $\beta$. Typically, for the Sun, this linewidth is of

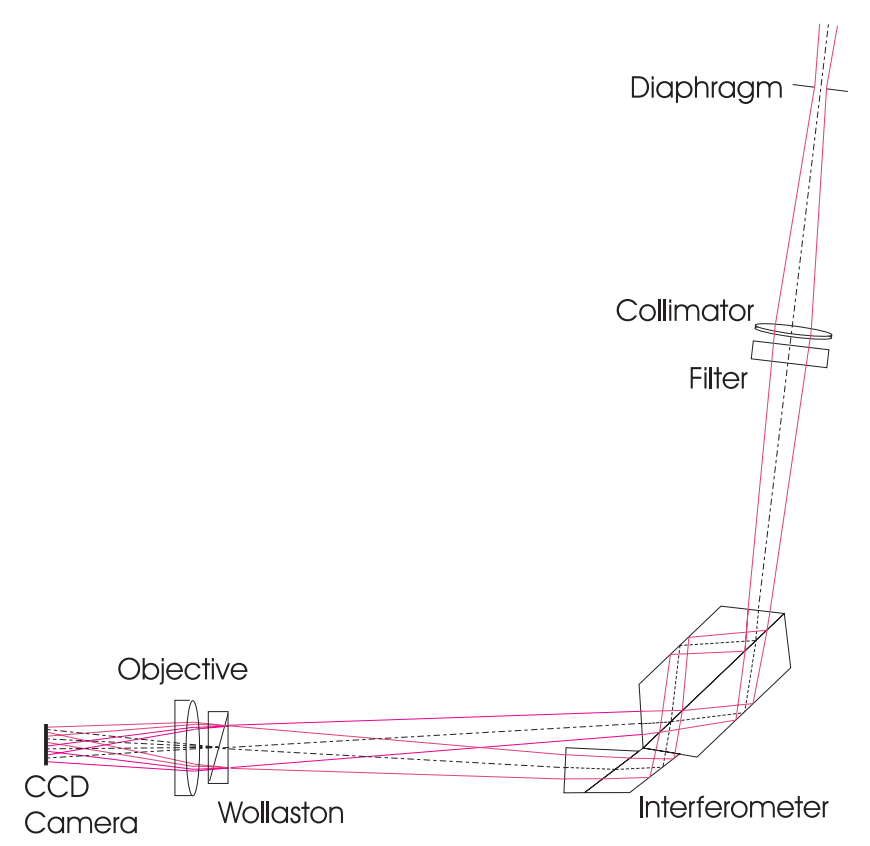

Fig. 4. Optical scheme of the instrument SYMPA. The Mach-Zehnder interferometer is made of two glass blocks glued together with an intensity separation in between. A $5 \mathrm{~nm}$ bandwidth is selected by an interference filter. The Wollaston polarizer separates each output from the interferometric device in two separated beams. In total, the instrument produces four images of the same field on the camera, separated by $\pi / 2$ in phase.

the order of $1 \mathrm{~cm}^{-1}$, so a maximum of sensitivity is obtained for OPDs of the order of $1 \mathrm{~cm}$.

In the chosen bandwidth around the magnesium triplet at $517 \mathrm{~nm}$, we found a clear maximum of the sensitivity at $1.24 \mathrm{~cm}$ OPD (Fig. 1), for a relatively large bandwidth (up to $5 \mathrm{~nm}$ ). A complete exploration of the solar spectrum can be found in Mosser et al. (2003). Although other bandwidths at shorter wavelengths may provide a better sensitivity, the choice of this maximum at $517 \mathrm{~nm}$ ensures a good compromise with detector sensitivity (maximum in the green), seeing quality (as it decreases with $\left.\lambda^{\frac{6}{5}}\right)$, and instrumental requirements, also more stringent for shorter wavelengths.

Actually, the Doppler effect is doubled because the solar light is reflected on the moving surface of Jupiter. Finally, the fringe pattern is obtained by

$$
\begin{aligned}
I(\Delta) & =\left|\int S_{\mathrm{p}}(\sigma) \mathrm{e}^{2 \pi \sigma \Delta} \mathrm{d} \sigma\right|^{2} \\
& \simeq I_{0}\left[1+C(\Delta) \cos \left(2 \pi \sigma_{0} \Delta\left(1+2 \frac{\delta v}{c}\right)\right)\right] .
\end{aligned}
$$

With our configuration, the phase sensitivity is approximatively:

$\frac{\delta \varphi}{\delta v} \simeq 1 \mathrm{rd} / \mathrm{km} \mathrm{s}^{-1}$.

In order to minimize thermal and pressure variations of the OPD, and to be able to use both opposite outputs, a compact Mach-Zehnder design was chosen for the interferometer. Its optical design is displayed in Fig. 4. With such a compact glass block, an efficient thermal stability is easily obtained. Moreover, it is made of two different glasses in order to minimize the thermal dependence of the OPD. This point will be addressed in the next paragraph. 


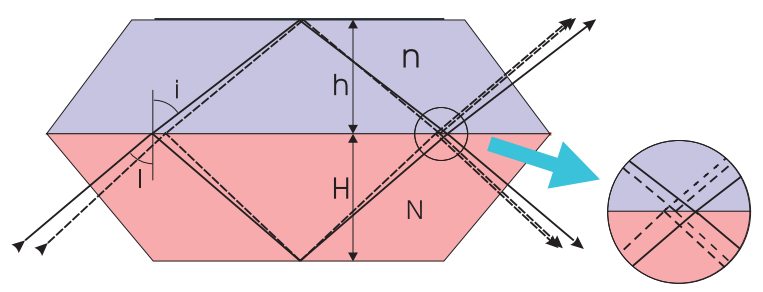

Fig. 5. Detail of the optical path within the prism. On one arm, the light is totally reflected. On the other arm, a metallic reflection occurs. The OPD depends on the incoming angle, as shown on the detail. For a nominal input, plain line, the OPD is almost stationary.

In the Mach-Zehnder design, the OPD can be written as

$$
\begin{aligned}
\Delta\left(A_{x}, A_{y}, \lambda\right) & =2 H N(\lambda) \cos \left(A_{x}\right) \cos \left(A_{y}\right) \\
& -2 h n(\lambda) \sqrt{1-\left[\frac{N(\lambda)}{n(\lambda)} \sin \left(A_{x}\right) \cos \left(A_{y}\right)\right]^{2}}
\end{aligned}
$$

where $A_{x}$ and $A_{y}$ are the angles at the entrance of the optical block, respectively in the horizontal and vertical directions. This produces the two dimensional image of the fringes (Fig. 6). It can be seen that the angular variation on the vertical axis is negligible. The vertical fringe pattern is a slowly changing period, which becomes almost stationary on the right part of the field. In Fig. 7, we see that the contrast of the fringes remains almost uniform along the field.

\subsection{Chromatic effects}

Due to the chromatic dependence of the OPD and its effect on the interference pattern, the actual optimal OPD at the central wavelength is not exactly the one which has been previously calculated. Indeed, the interference calculated for the Mach-Zehnder interferometer

$I\left(A_{x}, A_{y}\right)=\int S_{\mathrm{p}}(\sigma) \mathrm{e}^{2 \pi \mathrm{i} \Delta\left(A_{x}, A_{y}, \sigma\right) \sigma} \mathrm{d} \sigma$

is not anymore a pure Fourier transform of the spectrum expressed in $\sigma$, but a harmonic analysis of the same spectrum with a varying frequency. A development at the first order of Eq. (8) yields the expression:

$I\left(A_{x}, A_{y}\right) \simeq I_{0}\left[1+C\left(\Delta_{0}+\frac{\delta \Delta}{\delta \sigma} \sigma_{0}\right) \cos \left(2 \pi \sigma_{0} \Delta_{0}\left(A_{x}, A_{y}\right)\right)\right]$.

By analogy with Eq. (1), the previously found maximum of contrast at an OPD of $1.244 \mathrm{~cm}$ in the air is reached by adapting the height of the two pieces in such a way that $\left(\Delta+\frac{\delta \Delta}{\delta \sigma} \sigma_{0}\right)$ corresponds to this value of $1.244 \mathrm{~cm}$. In this case, the new geometrical OPD in the glass is $1.060 \mathrm{~cm}$. Figure 7 shows that the maximum contrast is obtained near the center of the field, but with a slightly reduced contrast value, because of this chromatic effect.

\subsection{Noise sources}

In order to estimate the instrumental capability for detection of small velocity variations, we should list all the possible sources of noise, and determine their levels.

Obviously the first - and unavoidable - source of noise is the photon shot noise. A precise estimation of it could be obtained, with several assumptions about the transmissions of the different parts of the instrument, which will be easily checked. However, we have to take into account, as much as possible, all other undesired mechanisms and estimate their contributions, compared to the photon noise, in the frequency domain where we seek planetary oscillations.

It is easy to determine three different contributions in the final noise level: the atmospheric effects, the telescope movements, and the instrumental noises. Here, we do not regard the solar and Jovian effects on the light as a noise source, even for those which could deteriorate the oscillations background, but as a part of the astronomical measurement. The arguments about the detectability of the oscillations will be addressed in a further paper.

The atmospheric contribution has little effect on the noise level in the given frequency range: indeed, atmospheric turbulence is much faster than this, and its main effect is to filter the high angular frequencies, limiting the accessible mode degree. A typically good seeing of $1^{\prime \prime}$ would allow the detection of modes up to the degree $\ell=25$ on Jupiter. However this value is not always reached, so the modes that we expect to follow with a good temporal coverage do not exceed degree between 10 and 15 on Jupiter, less than 8 on Saturn. In the conclusion, we will examine the possibilities to improve these numbers.

The final contribution in term of velocity precision is related to the way the images are formed, and how we combine them to reconstruct the velocity field. In particular, we should remind that the velocity is derived from the phase of the interference pattern, which is obtained by combining four images obtained at the same time. The improvement of the signal/noise statistic with the time is only obtained if the phase dispersion for a given measurement is well below $2 \pi$ at each point of the image. This condition has to be checked in the first place.

\subsubsection{Photon noise}

The phase, and therefore, the velocity map, is obtained from intensity measurement in four points along the fringe, as described in Fig. 3. It is easy to demonstrate (see Fig. 8) that the phase variance due to photon noise $\delta \varphi$ can be written as

$$
\begin{aligned}
\langle\delta \varphi\rangle^{2} & =\frac{\sigma_{X}^{2}+\sigma_{Y}^{2}}{X^{2}+Y^{2}} \\
& =\frac{2}{\gamma^{2} \mathcal{N}_{\mathrm{T}}}
\end{aligned}
$$

where $\mathcal{N}_{\mathrm{T}}$ is the total number of collected photons, i.e. about 4 times the photon number in each channel $\mathcal{N}_{0}$, and $\gamma$ the contrast of the fringes. This comes from the fact that only the spectral lines in the entrance bandwidth contribute to the signal, resulting in a low contrast, when the noise comes from the whole number of photons in the entrance filter.

Then, the standard deviation on the velocity variations, due to photon noise, can be estimated by

$$
\delta v=\frac{c}{2 \pi \sigma_{0} \Delta \gamma \sqrt{2 \mathcal{N}_{\mathrm{T}}}}
$$

Atmospheric transmission $\tau_{a}$ has been fixed at $70 \%$ in average, which is a rather pessimistic value for a high altitude observatory. Using the instrumental transmission calculated in Table 1, the total number of photons received on the detector from Jupiter at its maximum of brightness should reach $4 \times 10^{8}$ photons per second, for a $1.5 \mathrm{~m}$ telescope. The photon noise produces a standard deviation of $8 \mathrm{mrad}$ in one second, corresponding 

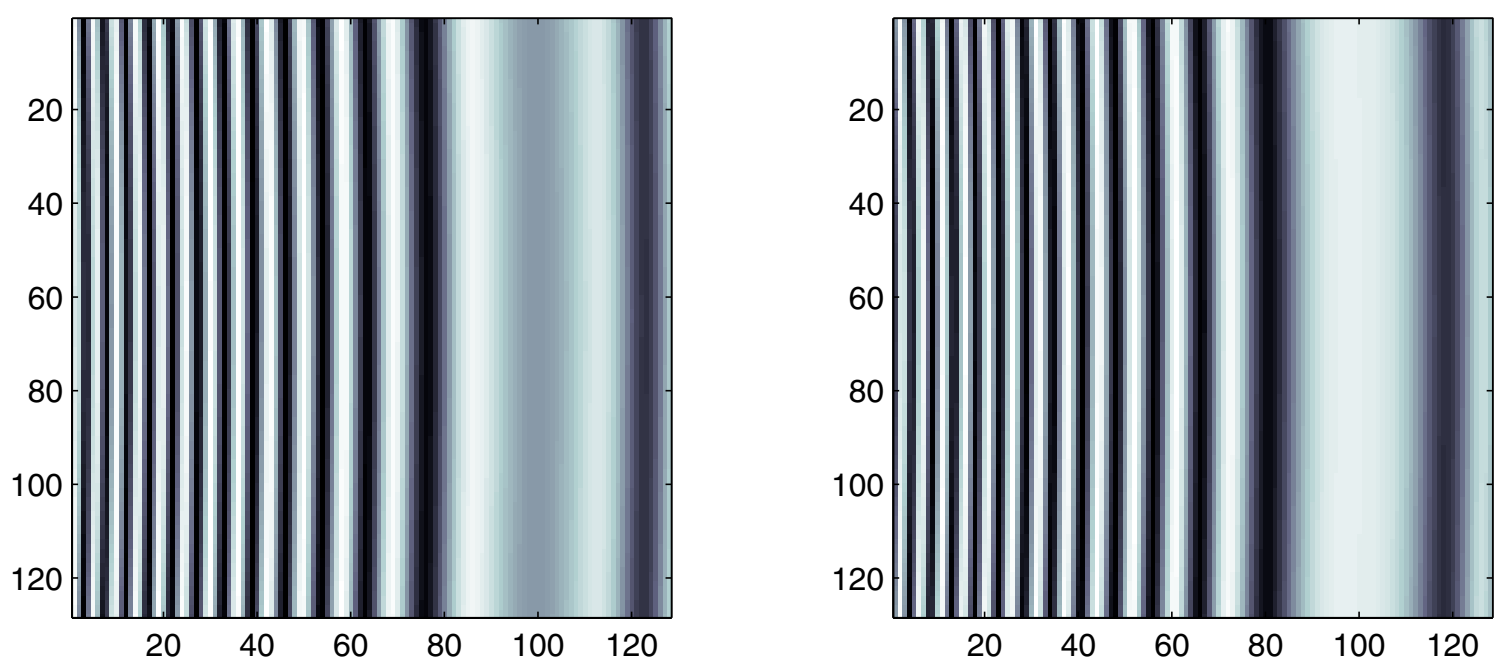

Fig. 6. Theoretical instrumental interference patterns for both polarizations as seen on the camera, with a total field of 1.3 arcmin on the sky (128 pixels). The maximum size of Jupiter is 48 arcsec, corresponding to 80 pixels on the camera.

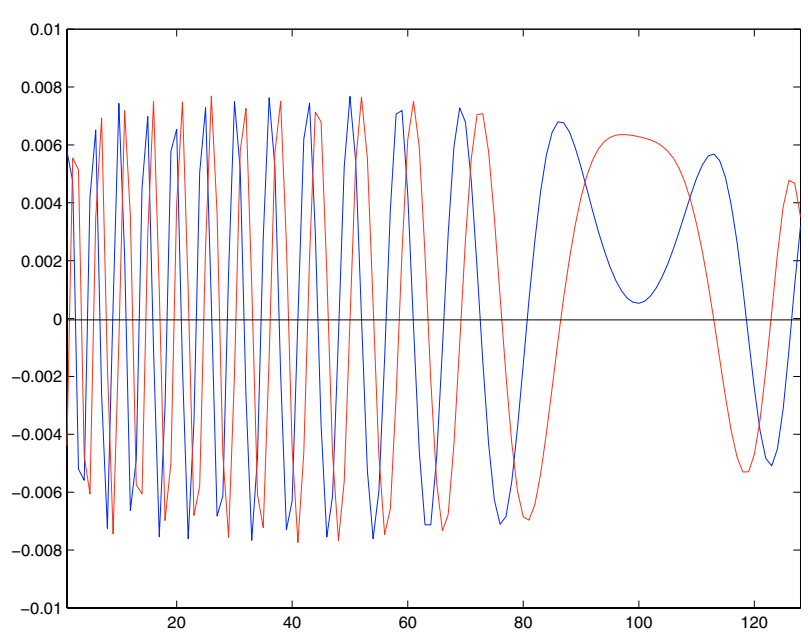

Fig. 7. Cut of the previous figure showing the contrast of the fringes along the field. The contrast is of the order of $0.7 \%$ in the middle of the field, and remains almost uniform on the central field of 80 pixels, corresponding to the size of Jupiter.

to a velocity noise of $8 \mathrm{~m} \mathrm{~s}^{-1}$ on the whole surface of Jupiter. The expected noise level is therefore of $5 \mathrm{~cm} \mathrm{~s}^{-1}$ after $8 \mathrm{~h}$ of observations.

This is a great improvement with respect to previous observations, where the theoretical performances were at least 10 times worst. The main improvement here comes from the spatial resolution of the instrument. Indeed, the fast rotation of Jupiter was a major cause of degradation of the sensitivity for the previous full-disk Doppler shift measurements. Another gain comes from the improvement in the detector efficiency.

\subsubsection{Thermal variations}

The advantage of the interferential spectrometer is to permit a high spectrometric resolution in a small and compact design, and also a full spatial resolution. Thanks to this compact design, the temporal variations of the interferometer response can be reduced, in particular the thermal dependency of the OPD. However, it would be much higher than the required stability, if we would not have taken special care in the instrumental design

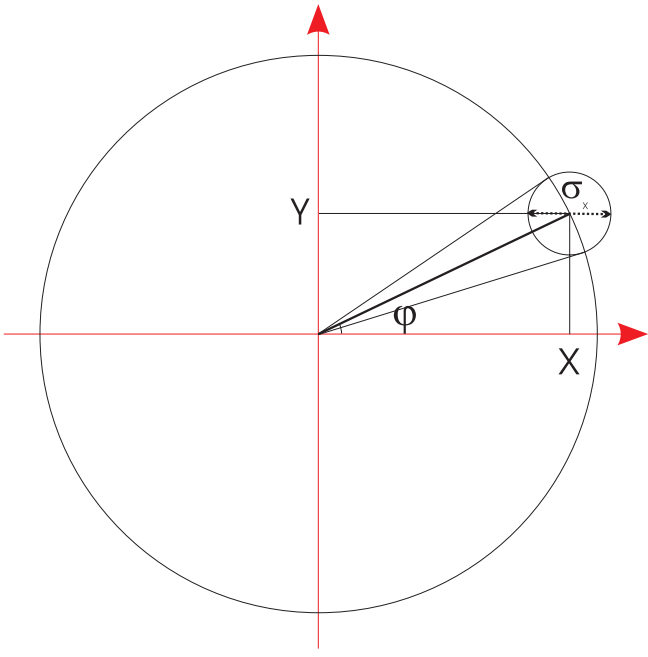

Fig. 8. Standard deviation on the phase determination, as obtained by the formula in Eq. (4), with $\mathrm{X}=(\mathrm{A}-\mathrm{C}) /(\mathrm{A}+\mathrm{C})$ and $\mathrm{Y}=(\mathrm{B}-$ D) $/(B+D)$.

Table 1. Efficiency of the instrument.

\begin{tabular}{l|c}
\hline \hline & Transmission \\
\hline Atmosphere & $70 \%$ \\
Telescope & $90 \%$ \\
Entrance Filter on 5 nm bandwidth & $45 \%$ \\
Lenses + Prism + Mirror & $85 \%$ \\
CCD Quantum Efficiency (@517 nm) & $85 \%$ \\
Camera duty-cycle (read-out time) & $75 \%$ \\
\hline Total & $15 \%$ \\
\hline
\end{tabular}

to minimize the thermal effects. The interferometer is made of two pieces of two different glasses, specially chosen as to compensate the index variations and the dilatation, in order to have a stable OPD.

Figure 9 shows the effects of temperature on velocity measurements. The instrument has been designed to minimise these effects precisely in the considered bandwidth. The best compensation is obtained for a temperature of $14{ }^{\circ} \mathrm{C}$, where the thermal variation is zero at the center of the field. At most, within a working range of $10{ }^{\circ} \mathrm{C}$, the maximal velocity variation for 

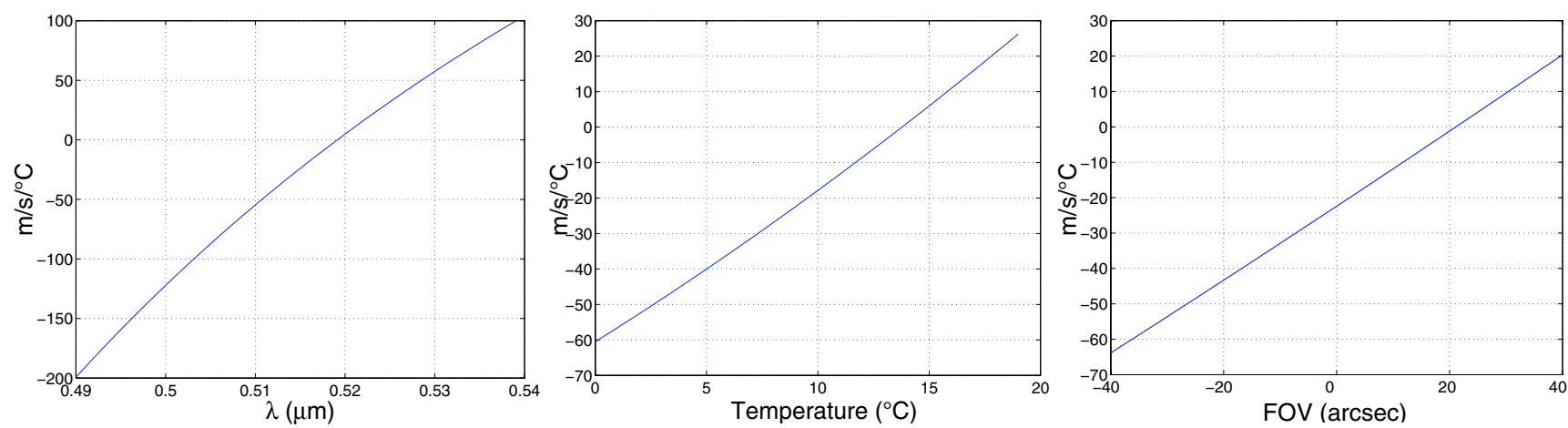

Fig. 9. This figure shows the effect of the temperature on the velocity measurement variations. On the left, we show this effect for different wavelengths: the materials and heights of the prisms have been chosen to minimise this effect at the central wavelength of our filter, at $517 \mathrm{~nm}$. The central figure shows the thermal dependance of the velocity measurement as a function of the temperature, showing a minimum effect for an external temperature of $14^{\circ}$. Right, the thermal variations along the field of view for a variation of one degree around $10^{\circ} \mathrm{C}$.

$1{ }^{\circ} \mathrm{C}$ is $60 \mathrm{~m} \mathrm{~s}^{-1}$ at one side of the field. The thermal variation of the filter wavelength also has to be taken into account, but it has been shown that its effect is less than $10 \%$ of the total thermal variation.

This sensitivity is small enough so it is possible to let the temperature vary in the instrument, with a passive thermal insulation to minimise the fast variations. With a temperature drift of the order of $1{ }^{\circ} \mathrm{C}$ in $8 \mathrm{~h}$ (typical of night conditions), the induced signal will be of the order of 20 to $40 \mathrm{~m} \mathrm{~s}^{-1}$, depending of the external temperature. This can be corrected easily, with a measurement of the prism temperature.

\section{Laboratory measurements}

The previous calculations assumed a perfect instrument. The actual interferometer is not perfect. First, it was difficult to ensure a constant and accurate OPD when gluing both prisms constituting the Mach-Zehnder interferometer. This problem was circumvented by placing the beam splitter treatment on one of the prisms for the separation and on the other prism for the recombination, so that the glue width does not affect the OPD. Another problem, more difficult to solve, was the possibility to achieve a perfect beam splitter, with equal properties for both polarizations. The problem was complicated by the fact that the two prisms were made of different glasses and then the beam splitter was not applied on the same material. Despite several tests, a satisfactory solution has not been encountered. The actual beam splitter achieves a transmission and reflection of 50\% and 50\% for one of the polarizations, but $30 \%$ and $70 \%$ for the other. This decreases the contrast on one of the components. Moreover, although the theoretical phase shift induced by a beam splitter is supposed to be $90^{\circ}$, actual phase difference of the interference fringes between both polarizations is about $62^{\circ}$ (see Fig. 10). The instrument exhibits also a global polarization effect, resulting in a better transmission of one channel with respect to the other.

These discrepancies between actual and theoretical expectations lead to an increase of the noise level, which has to be estimated. If $\theta$ is the phase shift between the two estimates $X$ and $Y$ in both polarizations, it is possible to build two new values $X^{\prime}$ and $Y^{\prime}$, defined by

$$
\begin{aligned}
X^{\prime} & =\frac{X \cos \frac{\theta}{2}-Y \sin \frac{\theta}{2}}{\cos \theta} \\
Y^{\prime} & =\frac{Y \cos \frac{\theta}{2}-X \sin \frac{\theta}{2}}{\cos \theta} .
\end{aligned}
$$

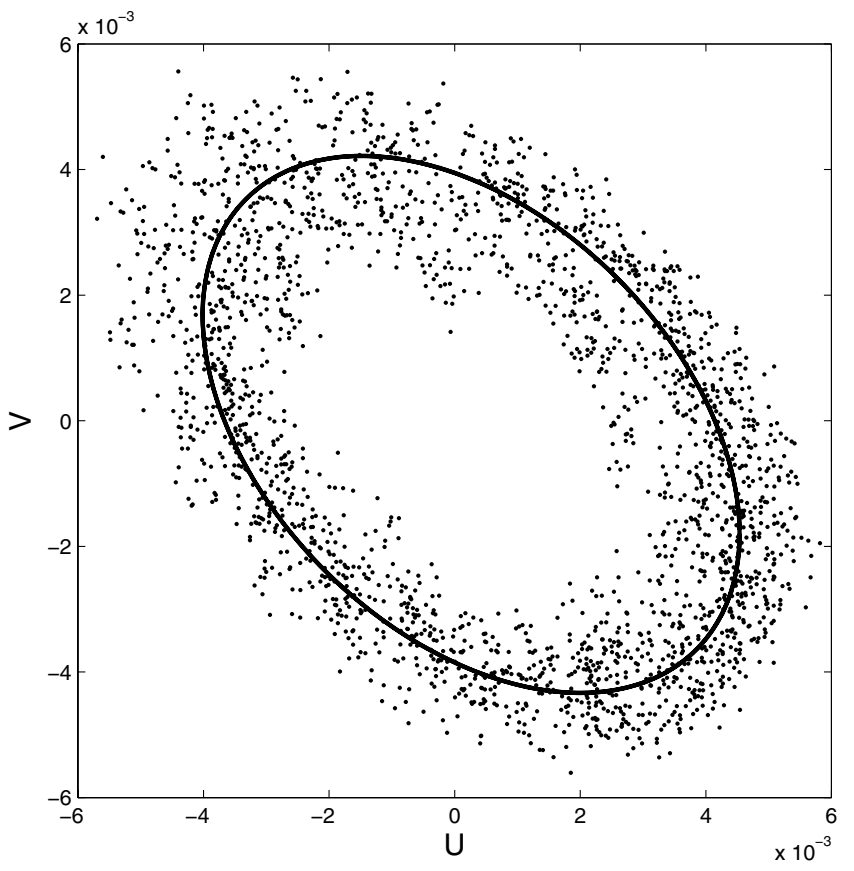

Fig. 10. Plot of one the interference pattern as a function of the other. It can be seen that both fringes are not in perfect quadrature. Note also the actual amplitude of the fringes obtained on the diffused solar light, as compared to the theoretical contrast.

These new variables are now in quadrature. Note that this linear combination between $X$ and $Y$ is not the only one which produces the correct value of $\varphi$, but this particular choice produces a uniform standard deviation at any phase, if the noise level is the same in both channels. The new standard deviation $\sigma_{\varphi}^{\prime}$ corresponding to the departure to quadrature can be written as

$\sigma_{\varphi}^{\prime}=\frac{\sigma_{\varphi}}{\cos (\theta)}$

In our case, the noise level is increased by $13 \%$, with respect to an interferometer in perfect quadrature.

A non-uniform photometric response in the different channels also leads to an increase of the photon noise contribution to the phase measurement. In the extreme case where one channel will receive no photon at all, the variance of the measurement will become infinite. If we consider the repartition of the incoming photons $\mathcal{N}_{\mathrm{T}}$ to be $\alpha \mathcal{N}_{\mathrm{T}}$ and $(1-\alpha) \mathcal{N}_{\mathrm{T}}$ between both polarizations, the variance of the phase measurement can be also 


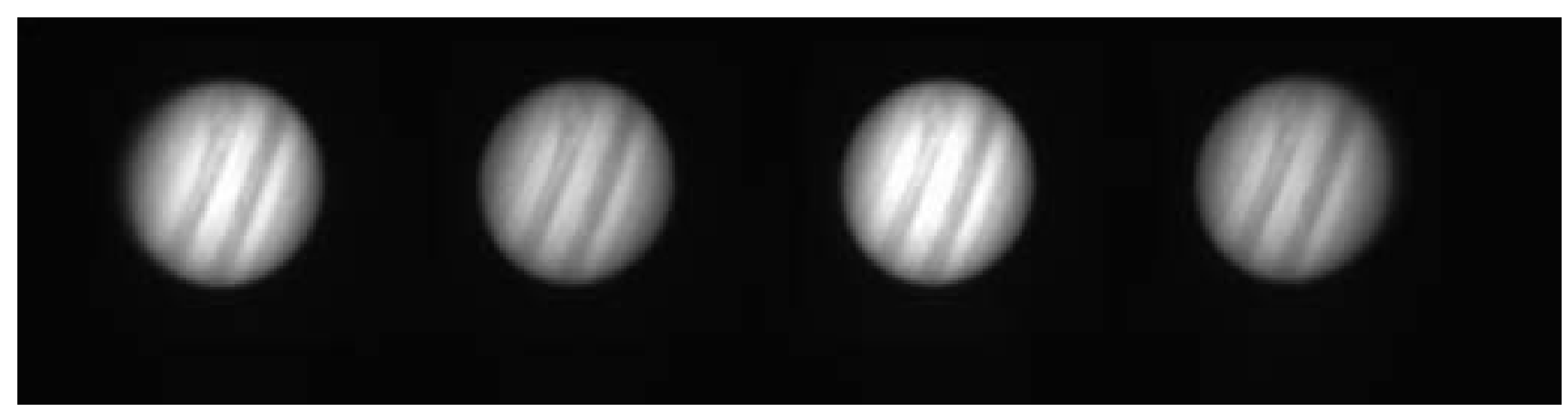

Fig. 11. Typical image of Jupiter obtained by SYMPA. The combination of the four images permits us to recover the phase of the interference pattern, a phase which senses the velocity field. The differences in the intensities are due to a non-perfect beam splitter. The fringes are invisible because of the too low contrast.

Table 2. Average efficiency of the 3 telescopes STEPHI network (from Hernandez Corujo Ph.D. Thesis).

\begin{tabular}{ccc}
\hline \hline $187 \mathrm{~h}$ & $37 \%$ & 1990 \\
$219 \mathrm{~h}$ & $46 \%$ & 1991 \\
$168 \mathrm{~h}$ & $39 \%$ & 1992 \\
$228 \mathrm{~h}$ & $46 \%$ & 1993 \\
$151 \mathrm{~h}$ & $35 \%$ & 1995 \\
$210 \mathrm{~h}$ & $31 \%$ & 1996 \\
$115 \mathrm{~h}$ & $21 \%$ & 1997 \\
\hline
\end{tabular}

calculated by the formula

$\sigma_{\varphi}^{\prime \prime}=\sigma_{\varphi} \sqrt{\frac{\alpha^{2}+(1-\alpha)^{2}}{\alpha(1-\alpha)}}$.

In our case, the transmission on one polarization is only $70 \%$ of the other, mainly due to the effect of the beam splitter. The corresponding augmentation of the noise level is of about $20 \%$.

But the main effect is the decrease of the contrast due to a non-uniform OPD along the pupil image within the prism. The contrast reaches only $4.5 \times 10^{-3}$ instead of the calculated value of $7 \times 10^{-3}$, increasing the noise level by about $30 \%$.

All these effects produce a degradation of the expected performances by a factor of 2 . In total, the expected white noise level for the actual instrument is about $10 \mathrm{~cm} \mathrm{~s}^{-1}$ in $8 \mathrm{~h}$ of observations of Jupiter, near the opposition, with a $1.5 \mathrm{~m}$ telescope. This value still allows a large improvement of performance with respect to previous observations. Taking into account the projection factor of a radial, or non-radial, oscillations on the lineof-sight, it would allow the detection of $50 \mathrm{~cm} \mathrm{~s}^{-1}$ amplitude oscillation with a signal-to-noise ratio of 3 .

\section{Observing strategy}

As said previously, discontinuities in the data was one of the main problems for mode identification in previous observations. To improve this situation, three strategies can be envisioned: space borne observations, ground-based observations at polar latitudes, or a network. The first two options will be examined in the conclusion. However, the simplest option to set-up for a first tentative is the following one: networks observations can generally be undertaken in the Northern hemisphere with a relatively good success.

Indeed, the STEPHI network, aiming to observations of $\delta$ Scuti stars, has proved to achieve an average duty cycle of $40 \%$
Table 3. Observatories data of the STEPHI network.

\begin{tabular}{c|ccc}
\hline \hline Observatory & Latitude & Longitude & Altitude \\
\hline Teide & +28.3 & $1.1 \mathrm{~h}$ & $2380 \mathrm{~m}$ \\
San Pedro Martir & +31.0 & $7.7 \mathrm{~h}$ & $2830 \mathrm{~m}$ \\
Xing-Long & +40.4 & $16.2 \mathrm{~h}$ & $870 \mathrm{~m}$ \\
\hline
\end{tabular}

with 3 telescopes for a typical observing period of 20 days, as shown in the previous table.

The same strategy is used for SYMPA. The instrument is easy to replicate and transport, and has the right efficiency for $1.5 \mathrm{~m}$ telescope. Three copies of the instrument were realised, allowing observations for two to three weeks, with a network similar to STEPHI, in San Pedro Martir, Izaña and Xing Long. If successful, such an observing run could reach about $50 \%$ duty cycle over 16 days. With the previous value for the performance, we expect to be able to detect oscillations of $10 \mathrm{~cm} \mathrm{~s}^{-1}$ in amplitude with a SNR of 3 .

\section{Conclusion}

It has been shown that the imaging interferometer designed for SYMPA is perfectly suitable for seismology of giant planets. In particular, in the case of Jupiter, a noise level lower than $4 \mathrm{~cm} \mathrm{~s}^{-1}$ can be expected. In any case, it will allow us to validate definitively the previous observations, where an amplitude of $50 \mathrm{~cm} \mathrm{~s}^{-1}$ to a few meter per second is found. It will also permit to detect and identify modes up to the degree $\ell=20$.

Observing Saturn with the same instrument would require a full network of three 2-m class telescopes to reach a noise level of about $10 \mathrm{~cm} \mathrm{~s}^{-1}$, for modes up to the degree $\ell=8$. It would also be highly interesting to observe oscillations on Neptune, but such observations requires larger telescopes ( $4 \mathrm{~m}$ telescope), and an adaptive optic system, if modes of degree higher than 3 are seek. Observations of Uranus appear unjustified; in absence of convection in this planet, no excitation mechanism of the oscillation is expected.

It has been shown that the SYMPA concept is able to improve the detection of oscillations on Jupiter and Saturn, both in signal to noise ratio and in number of detectable modes. However, it requires the use of a network of medium class telescopes. Experience of this type of observations has proved that a duty cycle higher than $50 \%$, for time series longer than 20 days, is very difficult to obtain.

The long term helioseismic observations (IRIS, GONG, SOHO) have shown that few week runs are generally insufficient to take the full reward from the oscillations measurement. 
However, observation runs longer than that are almost impossible to organize without a dedicated network. Another strategy is required, implying fully automatic measurements. Although such plans are developed for asteroseismology based on groundbased networks, it seems more efficient to develop a specially designed ground-based telescope in Antarctica or to have a devoted spatial instrument. Both solutions were considered: JOVIS in space (Baglin et al. 1999) and JISCO in Antarctica (Schmider et al. 2005). The Concordia station at Dome $C$ offers a unique opportunity to set-up a program for giant planets seismology, aiming to record oscillation with spatial resolution and taking advantage of the long polar night and the excellent site quality (Agabi et al. 2006; Aristidi et al. 2005). It has been shown (Schmider et al. 2005) that an instrument similar to SYMPA on a $40 \mathrm{~cm}$ telescope working continuously during the five months of the polar night will reach a better signal to noise ratio than a network of $1.5 \mathrm{~m}$ telescopes in three weeks. A duty cycle of more than $80 \%$ for 3 months can be expected. Such a project would increase the precision on the frequencies of the modes and the number of accessible modes by a factor of ten, at least, allowing much more detailed physical processes in the Jovian internal structure to be addressed. More recently, a similar instrument has been proposed for the seismology of Jupiter, aboard an interplanetary spacecraft, during the cruise phase of a mission to Europa and the Jupiter system (Blanc et al. 2006).

Acknowledgements. The authors are very grateful to the support offered by the team of the Laser-Lune 1.5-m telescope at the Observatoire de la Côte d'Azur. Most of the data have been obtained at the Observatorio San Pedro Martir, Baja California, operated by the Instituto de Astronomia de la UNAM. Many thanks to the support team of this observatory. These observations were partly supported by the CNRS-CONACYT agreement. Part of the data was also obtained at Carlos
Sanchez telescope at Izaña observatory, thanks to the help of the Instituto de Astrofisica de Canarias and partially supported by OPTICON Trans National Access program. The SYMPA instrument and the network were supported by the Programme National de Planétologie and the CNRS. The authors are grateful to the referee for useful comments.

\section{References}

Agabi, A., Aristidi, E., Azouit, M., et al. 2006, PASP, 118, 344 Aristidi, E., Agabi, K., Azouit, M., et al. 2005, A\&A, 430, 739 Baglin, A., Mosser, B., \& JOVIS Team 1999, AAS/Division for Planetary Sciences Meeting Abstracts, 31, \#08.13

Blanc, M., Coradini, A., Drossart, P., et al. 2006, European Planetary Science Congress 2006, 684

Deming, D., Mumma, M. J., Espenak, F., et al. 1989, ApJ, 343, 456

Gudkova, T., \& Zharkov, V. N. 1999, Planet. Space Sci., 47, 1211

Gudkova, T., Mosser, B., Provost, J., et al. 1995, A\&A, 303, 594

Guillot, T. 1999, Planet. Space Sci., 47, 1183

Guillot, T., Chabrier, G., Gautier, D., \& Morel, P. 1995, ApJ, 450, 463

Harvey, J. W., Hill, F., Hubbard, R., et al. 1996, Science, 272, 1284

Hernández Corujo, M. M. 1998, Universidad de la Laguna, Ph.D. Thesis

Maillard, J. P. 2000, ASP Conf. Ser., 195, 185

Maillard, J. P., \& Michel, G. 1982, IAU Coll., 67, ASSL, 92, 213

Michel, E., Chevreton, M., Belmonte J. A., et al. 2000, IAU Coll. 176, ASP Conf. Ser., 203, 483

Mosser, B., Gauhtier, D., Schmider, F. X., \& Delache, P. 1991, A\&A, 251, 356

Mosser, B., Mekarnia, D., Maillard, J.-P., et al. 1993, A\&A, 267, 604

Mosser, B., Maillard, J. P., \& Mékarnia, D. 2000, Icarus, 144, 104

Mosser, B., Maillard, J. P., \& Bouchy, F. 2003, PASP, 115, 990

Schmider, F.-X., Fossat, E., \& Mosser, B. 1991, A\&A, 248, 281

Schmider, F.-X., Gay, J., Jacob, C., et al. 2003, Ap\&SS, 284, 449

Schmider, F. X., Jacob, C., Fossat, E., et al. 2005, EAS Pub. Ser., ed. M. Giard, F. Casoli, \& F. Paletou, 285

Shao, M., \& Staelin, D. H. 1980, Appl. Opt., 19, 1519

Tomczyk, S., Streander, K., Card, G., et al. 1995, Sol. Phys., 159, 1

Vorontsov, S., Zharkov, V. N., \& Lubimov, V. M. 1976, Icarus, 27, 109 